\title{
Ausência de efeito antinociceptivo decorrente da administração intravenosa de crotalfina em comparação com morfina, U50-488H ou fenilbutazona em equinos submetidos à estimulação térmica da pele íntegra
}

\author{
Lake of antinociceptive effect caused by intravenous administration of crotalphine em \\ comparision to morphine, U50-488H or phenylbutazone in horses submitted to thermal \\ stimulation of the normal skin
}

\author{
Erica Cristina Bueno do Prado Guirro ${ }^{\mathrm{I}}$ João Henrique Perotta ${ }^{\mathrm{II}}$ Márcio de Paula ${ }^{\mathrm{III}}$ \\ Yara Cury $^{\mathrm{IV}}$ Carlos Augusto Araújo Valadão ${ }^{\mathrm{V}}$
}

RESUMO

Crotalfina é um novo peptídeo analgésico que atua em receptores opioides kappa e delta promovendo potente analgesia em ratos submetidos a modelos de dor inflamatória, neuropática ou oncológica. Talvez a crotalfina possa ser utilizada para tratar a dor em outras espécies. Assim, o objetivo deste estudo foi avaliar a resposta nociceptiva na região escapular e isquiática de cavalos tratados com crotalfina, morfina, U50$488 \mathrm{H}$ ou fenilbutazona e submetidos à estimulação térmica na pele íntegra. Dezoito cavalos da raça Puro Sangue Árabe foram alocados em cinco grupos experimentais: GC (5mL NaCl 0,9\%), GCRO (3,8mg. $\mathrm{kg}^{-1}$ crotalfina), GK (160 $\mu \mathrm{g} \cdot \mathrm{kg}^{-1}$ U50-488H), GM (0,1mg. $\mathrm{kg}^{-1}$ morfina) e GF (4,4mg. $\mathrm{kg}^{-1}$ fenilbutazona). Os animais foram submetidos ao modelo de dor inflamatória por meio de estimulação térmica $\left(140^{\circ} \mathrm{C}\right)$ e durante $24 \mathrm{~h}$ avaliou-se a latência para o reflexo do frêmito cutâneo na região escapular (LRFCesc) e isquiática (LRFCisq). O U50-488H apresentou efeito antinociceptivo na região isquiática por duas horas, porém, nos demais momentos do grupo GK, bem como nos grupos $G C$, GCRO, GM e GF, não foi observado efeito antinociceptivo, visto que a LRFCesc e a LRFCisq na pele íntegra de cavalos não aumentaram em 24 horas de avaliação. Portanto, a crotalfina, a morfina, o U50-488H e a fenilbutazona não produziram efeito antinociceptivo relevante em equinos submetidos à estimulação térmica em pele íntegra.

Palavras-chave: analgésicos opioides, crotalfina, equinos, estimulação térmica, kappa, nociceptividade.

\section{ABSTRACT}

Crotalphine is a novel analgesic peptide that acts on kappa and delta opioid receptors providing powerful analgesia in rats submitted to inflammatory, neuropathic or oncologic pain model. Maybe crotalphine can be used to treat pain in other species. So, the aim of this study was to evaluate nociceptive response at scapular and isquiatic region of horses treated with crotalphine, morphine, U50-488H or phenylbutazone and submitted to thermal stimulation in complete skin. Eighteen Arabian horses

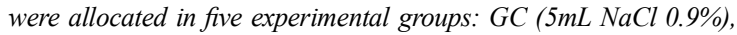
GCRO (3.8ng. $\mathrm{kg}^{-1}$ crotalphine), GK (160 $\left.\mu \mathrm{g} . \mathrm{kg}^{-1} \mathrm{U} 50-488 \mathrm{H}\right), \mathrm{GM}$ (0.1mg. $\mathrm{kg}^{-1}$ morphine) and GP (4.4mg. $\mathrm{kg}^{-1}$ phenylbutazone). Animals were submitted to inflammatory pain model by thermal stimulation $\left(140^{\circ} \mathrm{C}\right)$ and during $24 \mathrm{~h}$ latency to skin twitch at scapular and isquiatic region were evaluated. The U50-488H produced antinociceptive effect at isquiatic region along two hours, but, in other moments of $G K$ and in the other groups there was not antinociceptive effect, because LRFCesc and LRFCisq in complete skin of horses did not increase during $24 \mathrm{~h}$ evaluation. Thus, crotalphine, morphine, U50-488H and phenylbutazone did not cause relevant antinociceptive effect in horses submitted to thermal stimulation in complete skin.

Key words: crotalphine, horses, kappa, opioids, nociception, thermal stimulation.

\section{INTRODUÇÃO}

Os opioides são importantes analgésicos que atuam por diversos mecanismos após ligaremse aos receptores mu, kappa e delta (GAVERIAUXRUFF \& KIEFFER, 1999), principalmente reduzindo a liberação de neurotransmissores e,

ICurso de Medicina Veterinária, Universidade Federal do Paraná (UFPR), 85950-000, Palotina, PR, Brasil. E-mail: ericaguirro @ufpr.br. Autor para correspondência.

"Curso de Medicina Veterinária, Universidade Federal do Recôncavo Baiano (UFRB), Cruz das Almas, BA, Brasil.

${ }^{\mathrm{II} C}$ Consórcio da Indústria Farmacêutica (COINFAR), São Paulo, SP, Brasil.

${ }^{\mathrm{IV}}$ Laboratório de Patofisiologia, Instituto Butantan, São Paulo, SP, Brasil.

${ }^{\vee}$ Departamento de Clínica e Cirurgia Veterinária, Universidade Estadual Paulista (UNESP), Jaboticabal, SP, Brasil. 
consequentemente, reduzindo a transmissão neuronal (DICKENSON \& SULLIVAN, 1987; SCHULTZ \& GROSS, 2001).

A morfina é o padrão dos agonistas $\mathrm{mu}$ e a dose terapêutica para equinos varia de 0,04a $0,1 \mathrm{mg} \mathrm{kg}^{-1}$. Embora alguns autores relatem efeitos cardiorrespiratórios e aumento da atividade locomotora espontânea adversos (THURMON et al., 1996; MUIR et al., 2001), GUIRRO et al. (2010) consideram seguro o uso de $0,1 \mathrm{mg} \mathrm{kg}^{-1}$ de morfina em equinos. Buscando associar a analgesia dos opioides à menor incidência de efeitos adversos, pesquisas com agonistas kappa têm sido realizadas. O U50488 é considerado o padrão do grupo (DHAWAN et al., 1989) e o uso de $160 \mathrm{mg} / \mathrm{kg}$ em equinos induz analgesia potente com efeitos adversos discretos e irrelevantes (KAMERLING et al., 1988).

Segundo Vital Brazil relatou na primeira metade do século XX, vítimas de acidente crotálico não apresentavam reação inflamatória intensa e relatavam analgesia local (SOUSA \& SILVA et al., 1996). Posteriormente, identificou-se a presença de peptídeo opioide no veneno de Crotalus durissus terrificus e a aplicação deste em ratos promove analgesia significativa e prolongada em modelos de dor inflamatória(BRIGATTE et al., 2001), neuropática (GUTIERREZ, 2005) e oncológica (BRIGATTE et al., 2004). Mais recentemente, tal peptídeo opioide foi sintetizado e denominado crotalfina (GIORGI et al., 1993).

A crotalfina atua em receptores kappa centrais (PICOLO et al., 1998; BRIGATTE et al., 2001) e, perifericamente, atua em receptores kappa, delta e interfere na via óxido nítrico-GMPc L-arginina e nos canais K+ ATP-dependentes (PICOLO et al., 2000; PICOLO et al., 2003; PICOLO \& CURY, 2004). A administração de crotalfina em roedores produz analgesia efetiva e persistente, livre de efeitos adversos clínicos ou comportamentais (PICOLO et al., 2000; BRIGATTE et al., 2001; PICOLO \& CURY, 2004; NUNES et al., 2007) e, portanto, surge uma nova perspectiva em relação ao uso de crotalfina em outras espécies.

Os antiinflamatórios não esteroidais também são utilizados no tratamento da resposta inflamatória e da dor de origem inflamatória e a fenilbutazona na dose de 2,2 a $4,4 \mathrm{mg} \mathrm{kg}^{-1}$ é frequentemente prescrita para equinos (MAY, 1992).

Dessa maneira, o objetivo deste trabalho foi avaliar e comparar a resposta nociceptiva de equinos tratados com crotalfina, morfina, U50-488H ou fenilbutazona submetidos à estimulação térmica em pele íntegra.

\section{MATERIAL E MÉTODOS}

Dezoito equinos da raça Puro Sangue Árabe, sendo nove machos e nove fêmeas não prenhes, entre quatro e seis anos de idade, pesando $377 \pm 27 \mathrm{~kg}$, foram selecionados após exame físico, hemograma completo e avaliação da proteína total. Todos os animais passaram por 30 dias de adaptação à rotina experimental. Para evitar possíveis alterações, os ensaios experimentais foram iniciados sempre às $8 \mathrm{~h}$ e todo o estudo ocorreu entre setembro e outubro do mesmo ano.

Em cada equino, a região escapular e isquiática contra-lateral foram submetidas à tricotomia e utilizou-se tinta preta para pintar a pele dos animais para uniformizar a absorção e reflexão da luz, porém a temperatura da pele do animal não foi aferida. Estabeleceu-se um ponto central e foi desenhado um círculo ao redor, com $5 \mathrm{~cm}$ de raio, empregando-se tinta branca. A cauda dos animais foi enfaixada. Foi realizada a introdução de um cateter venoso $14 \mathrm{G}$ na veia jugular para administração dos fármacos a serem testados e os animais foram mantidos em repouso por 30 minutos.

Em TB (tempo basal) avaliou-se a latência para o reflexo do frêmito cutâneo na região escapular (LRFCesc) e isquiática (LRFCisq) a partir da aferição do tempo necessário para causar resposta aversiva à exposição de luz artificial de alta intensidade. Para tanto, empregou-se um processador para registrar o tempo de exposição a um foco de luz artificial ${ }^{a}$ com temperatura constante em $140^{\circ} \mathrm{C}$. Seguindo as recomendações do fabricante, para garantir foco único o feixe de luz foi aproximado do animal até que houvesse sobreposição de dois pontos luminosos, o que ocorria a cerca de $20 \mathrm{~cm}$ da pele. O foco foi direcionado ao ponto central previamente desenhado na pele até que o animal apresentasse frêmito cutâneo. Considerou-se 10 segundos como o tempo de corte para evitar queimaduras.

Após 20 minutos, foi realizada a injeção intravenosa dos fármacos conforme o grupo: $\mathrm{GC}(5 \mathrm{~mL}$ de $\mathrm{NaCl} 0,9 \%)$, GCRO (3,8mg. $\mathrm{kg}^{-1}$ de crotalfina), GM (0,1mg. $\mathrm{kg}^{-1}$ de morfina), GK (160 $\mu$ g. $\mathrm{kg}^{-1}$ de U50-488H) e GF (4,4mg. $\mathrm{kg}^{-1}$ de fenilbutazona), sendo o volume final ajustado em $5 \mathrm{~mL}$ utilizandose $\mathrm{NaCl}$ 0,9\%. Imediatamente verificou-se LRFCesc e LRFCisq e este foi considerado o momento T0. Reavaliações ocorreram em T1h, T2h, T3h, T6h, $\mathrm{T} 12 \mathrm{~h}$ e T24h. Tais avaliações foram realizadas sempre pelo mesmo avaliador oculto, que desconhecia o tratamento ao qual o animal havia sido submetido. 
Cada grupo experimental foi constituído por oito animais e todos os animais foram utilizados mais de uma vez. A distribuição dos animais foi parcialmente aleatória, pois cada grupo experimental deveria ter quatro machos e quatro fêmeas, não houve reutilização dos animais antes de 15 dias e nenhum animal foi utilizado duas vezes no mesmo grupo.

Quanto à análise estatística, utilizou-se Kruskal-Wallis, seguido pelo teste post hoc de Dunn $(p \leq 0,05)$ na comparação entre grupos; aplicou-se o teste Friedman em blocos com repetições múltiplas, seguido pelo teste de Dunnett $(\mathrm{P} \leq 0,05)$ na comparação entre tempos.

\section{RESULTADOS E DISCUSSÃO}

O U50-488H aumentou o LRFCisq entre T1h e T2h, porém não interferiu em LRFCesc durante todo o estudo. No grupo que recebeu $\mathrm{NaCl}$ $0,9 \%$ verificou-se aumento de LRFCesc em T12h e nenhuma alteração significativa em LRFCisq. Nos grupos tratados com morfina, crotalfina ou fenilbutazona não houve alteração em LRFCesc e de LRFCisq, conforme exibe a tabela 1.

Os resultados demonstram que os opioides utilizados nos equinos foram pouco eficientes para produzir efeito antinociceptivo em tecido não lesionado. Isso corrobora STEIN (1993) e ANTONIJEVIC et al. (1995) que inferem que a ação antinociceptiva dos agonistas opioides é pronunciada no tecido inflamado, mas pouco evidente em tecidos íntegros em diversas espécies animais. O sistema opioidérgico é eficiente para modular a resposta nociceptiva no tecido inflamado (TRUONG et al., 2003) conforme a natureza do estímulo e o estágio da reação inflamatória tecidual (MILLAN, 1986) que podem ativar os receptores opioides periféricos (STEIN \& LANG, 2009). Também nos tecidos periféricos é necessário haver inflamação tecidual para que a morfina promova adequado efeito analgésico (LIKAR et al., 2001). A aplicação do estímulo térmico sobre o mesmo ponto poderia ter favorecido a instalação de hiperalgesia (LEMONICA \& PEREIRA, 1992) e, portanto, foi importante estabelecer o tempo de corte ao estímulo térmico para evitar dano tecidual.

$\mathrm{Na}$ maioria dos estudos com equinos em que se verificou que os opioides produziram ação analgésica precoce e com duração de moderada a prolongada, utilizou-se modelo experimental em que havia injúria tecidual prévia (KALPRAVIDH et al., 1984; KAMERLING et al., 1988; BOATWRIGHT et al., 1996; SKARDA \& MUIR III, 2003). Como no presente estudo não havia inflamação prévia, os opioides não foram efetivos em interferir no limiar nociceptivo frente à estimulação térmica.

Os antiinflamatórios não esteroidais atuam na cascata do ácido aracdônico e na síntese de prostaglandinas (MITTEN \& HINCHCLIFF, 1997). Portanto, a ausência de inflamação tecidual prévia tornou a fenilbutazona ineficaz em interferir no limiar nociceptivo

O método de avaliação do efeito analgésico de um fármaco a partir da mensuração do tempo de latência para desencadear o frêmito cutâneo em equinos já foi utilizado em pôneis (PIPPI et al., 1979) e em equinos (KAMERLING et al., 1985). Ao estudar a analgesia da buprenorfina em equinos, CARREGARO et al. (2007) inferem que tal método foi o mais sensível. Ao empregar a mesma metodologia, DHANJAL et al. (2009) observaram que o tramadol não aumentou o limiar à estimulação térmica no tecido íntegro de equinos.

Geralmente, nos estudos com equinos, a latência necessária para provocar o frêmito cutâneo frente a uma temperatura constante e conhecida é observada na cernelha (KAMERLING et al., 1985) e na região dorsal da articulação escapuloumeral (CARREGARO et al., 2007). No presente estudo, além da região escapular utilizou-se a região isquitática visto alguns animais receberam injeção de agonista kappa e a distribuição espinhal de receptores opioide kappa restringe-se ao segmento lombosacro (SINATRA, 1993). Outra questão importante é o período de avaliação, pois KAMERLING et al. (1985) inferem que após 15 horas de avaliação pode ocorrer aumento da latência possivelmente decorrente da variação na liberação de opioides endógenos conforme o ciclo circadiano. No presente trabalho, houve avaliação em T24h, porém desde as primeiras aferições já não havia sido observado efeito antinociceptivo significativo. Portanto, mesmo que se excluam as avaliações na 24 a hora, o resultado deste estudo não será alterado.

A dose de crotalfina utilizada no GCRO foi calculada a partir da extrapolação alométrica da dose de crotalfina administrada com sucesso na analgesia de ratos (PICOLO \& CURY, 2004). Um prévio estudo (GUIRRO, 2008) verificou que a dose de $3,8 \mathrm{mg} \cdot \mathrm{kg}^{-1} \mathrm{de}$ crotalfina em equinos não alterou a atividade locomotora espontânea, a micção e a defecação dos animais e gerou apenas eventuais sinais estereotipados como bocejos e mastigação na primeira hora após a aplicação do fármaco, provavelmente decorrentes da menor liberação de dopamina causada pelo uso dos opioides agonistas de receptores kappa (DUKE et al., 1997). 
Tabela 1 - Mediana, valor do $1^{\circ}$ quartil, $3^{\circ}$ quartil, valor mínimo e máximo da latência para o reflexo do frêmito cutâneo na região escapular (LRFCese) e isquiática (LRFCisq) observada na pele íntegra de equinos submetidos aos grupos GC (5mL NaCl 0,9\%), GCRO

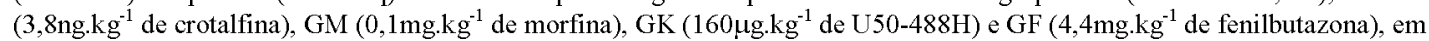
segundos.

\begin{tabular}{|c|c|c|c|c|c|c|c|c|c|}
\hline & & $\mathrm{TB}$ & T0 & $\mathrm{T} 1 \mathrm{~h}$ & $\mathrm{~T} 2 \mathrm{~h}$ & T3h & T6h & $\mathrm{T} 12 \mathrm{~h}$ & $\mathrm{~T} 24 \mathrm{~h}$ \\
\hline \multirow{3}{*}{$\mathrm{GC}$} & mediana & 2,6 & 2,5 & 2,6 & $2,8^{*}$ & 3,0 & 3,3 & 4,6 & 3,0 \\
\hline & $1^{\frac{9}{2}} 3^{o}$ quartil & $2,3 / 3,6$ & $1,9 / 3,2$ & $2,2 / 3,8$ & $2,4 / 3,3$ & $2,5 / 4,0$ & $2,2 / 5,5$ & $3,1 / 5,6$ & $2,5 / 3,6$ \\
\hline & mín / máx & $1,7 / 9,5$ & $1,6 / 10$ & $1,7 / 6,9$ & $1,6 / 6,8$ & $1,9 / 5,4$ & $1,6 / 7,3$ & $1,8 / 8,8$ & $2,0 / 10$ \\
\hline \multirow{3}{*}{ GCRO } & mediana & 2,3 & 2,9 & 2,5 & 2,9 & 3,0 & 3,6 & 2,5 & 3,5 \\
\hline & $1^{0} / 3^{\circ}$ quartil & $1,7 / 4,0$ & $1,8 / 5,0$ & $2,0 / 3,7$ & $2,3 / 4,3$ & $2,0 / 4,9$ & $2,7 / 5,3$ & $2,1 / 3,5$ & $2,1 / 4,4$ \\
\hline & mín / máx & $1,2 / 10$ & $1,5 / 7,3$ & $1,4 / 7,3$ & $1,0 / 6,9$ & $1,2 / 7,6$ & $1,7 / 10$ & $1,5 / 6,8$ & $0,9 / 10$ \\
\hline \multirow{3}{*}{ GM } & mediana & 3,1 & 2,8 & 4,1 & 3,7 & 2,8 & 3,8 & 5,3 & 3,4 \\
\hline & $1^{\mathrm{Q}} / 3^{\mathrm{Q}}$ quartil & $2,0 / 7,0$ & $2,1 / 5,0$ & $2,9 / 5,0$ & $2,7 / 4,9$ & $2,2 / 4,7$ & $2,7 / 6,9$ & $3,1 / 9,3$ & $2,9 / 6,1$ \\
\hline & min / máx & $1,2 / 8,5$ & $0,8 / 6,4$ & $1,5 / 9,8$ & $1,8 / 10$ & $1,5 / 10$ & $1,2 / 10$ & $1,0 / 10$ & $1,1 / 10$ \\
\hline \multirow{3}{*}{ GK } & mediana & 4,3 & 3,1 & 4,8 & 3,2 & 4,5 & 3,2 & 3,5 & 3,4 \\
\hline & $1^{\mathrm{o}} / 3^{\mathrm{o}}$ quartil & $2,1 / 6,6$ & $2,1 / 8,9$ & $2,6 / 5,9$ & $2,2 / 8,4$ & $3,0 / 10$ & $2,9 / 6,6$ & $3,0 / 4,6$ & $2,5 / 4,9$ \\
\hline & mín / máx & $1,0 / 10$ & $1,4 / 10$ & $1,5 / 10$ & $1,5 / 10$ & $1,6 / 10$ & $2,5 / 10$ & $1,1 / 10$ & $1,8 / 10$ \\
\hline \multirow{3}{*}{ GF } & mediana & 2,5 & 2,9 & 3,0 & 3,5 & 2,9 & 2,9 & 3,2 & 3,4 \\
\hline & $1^{\mathrm{o}} / 3^{\mathrm{o}}$ quartil & $2,3 / 4,2$ & $2,3 / 3,5$ & $2,6 / 3,9$ & $2,4 / 5,8$ & $2,0 / 4,0$ & $2,0 / 3,8$ & $2,4 / 3,9$ & $2,8 / 4,7$ \\
\hline & mín / máx & $1,2 / 10$ & $2,2 / 7,7$ & $1,3 / 5,6$ & $1,0 / 10$ & $1,4 / 10$ & $1,3 / 9,7$ & $1,1 / 10$ & $1,2 / 9,0$ \\
\hline \multirow{3}{*}{$\mathrm{GC}$} & mediana & 4,3 & 4,6 & 4,0 & 4,6 & 3,5 & 5,2 & 4,5 & 5,0 \\
\hline & $1^{\mathrm{Q}} / 3^{\mathrm{o}}$ quartil & $3,2 / 7,1$ & $3,7 / 8,6$ & $2,9 / 6,2$ & $3,4 / 6,6$ & $2,9 / 5,9$ & $4,0 / 10$ & $3,5 / 8,1$ & $3,5 / 6,6$ \\
\hline & min / máx & $1,8 / 10$ & $2,2 / 10$ & $2,0 / 10$ & $2,5 / 10$ & $2,3 / 10$ & $2,7 / 10$ & $2,3 / 10$ & $1,8 / 10$ \\
\hline \multirow{3}{*}{ GCRO } & mediana & 3,8 & 4,3 & 4,0 & 3,9 & 3,6 & 3,1 & 3,6 & 4,1 \\
\hline & $1^{9} / 3^{0}$ quartil & $2,7 / 6,9$ & $2,1 / 4,8$ & $2,5 / 8,0$ & $2,3 / 5,0$ & $2,4 / 6,3$ & $2,5 / 6,3$ & $3,0 / 4,8$ & $3,1 / 9,6$ \\
\hline & mín / máx & $2,0 / 10$ & $1,0 / 10$ & $1,2 / 10$ & $0,9 / 7,3$ & $1,6 / 9,8$ & $1,8 / 10$ & $2,1 / 10$ & $2,2 / 10$ \\
\hline \multirow{3}{*}{ GM } & mediana & 4,6 & 4,7 & 5,8 & 4,6 & 4,3 & 4,7 & 4,6 & 4,9 \\
\hline & $1^{o} / 3^{o}$ quartil & $3,8 / 7,3$ & $4,0 / 5,7$ & $4,0 / 7,9$ & $2,8 / 5,4$ & $3,5 / 6,7$ & $3,3 / 9,6$ & $2,9 / 5,9$ & $3,1 / 6,8$ \\
\hline & mín / máx & $2,5 / 10$ & $1,4 / 10$ & $2,7 / 10$ & $1,3 / 7,4$ & $1,2 / 10$ & $2,1 / 10$ & $2,4 / 10$ & $1,6 / 10$ \\
\hline \multirow{3}{*}{ GK } & Mediana & 5,4 & 4,2 & $7,5^{* \mathrm{ab}}$ & $9,9^{* \text { ab }}$ & 6,9 & 5,1 & 5,6 & 7,5 \\
\hline & $1^{9} / 3^{9}$ quartil & $3,5 / 8,4$ & $2,9 / 8,9$ & $4,2 / 9,9$ & $7,9 / 10$ & $5,4 / 10$ & $3,4 / 9,4$ & $3,2 / 7,5$ & $4,3 / 9,8$ \\
\hline & min / máx & $2,3 / 10$ & $2,4 / 10$ & $2,7 / 10$ & $3,3 / 10$ & $2,8 / 10$ & $1,5 / 10$ & $2,6 / 10$ & $3,0 / 10$ \\
\hline \multirow{3}{*}{ GF } & Mediana & 3,2 & 2,8 & 2,9 & 3,2 & 2,9 & 3,6 & 3,7 & 3,3 \\
\hline & $1^{\mathrm{o}} / 3^{\mathrm{o}}$ quartil & $2,5 / 4,9$ & $2,5 / 4,2$ & $2,2 / 4,8$ & $2,2 / 4,6$ & $2,1 / 4,5$ & $2,3 / 5,1$ & $3,1 / 6,1$ & $2,5 / 5,7$ \\
\hline & mín / máx & $1,7 / 10$ & $1,6 / 10$ & $1,2 / 7,6$ & $1,4 / 10$ & $1,4 / 10$ & $1,1 / 10$ & $1,3 / 10$ & $2,0 / 10$ \\
\hline
\end{tabular}

* diferente de TB; ${ }^{\mathrm{a}}$ diferente de $\mathrm{GC} ;{ }^{\mathrm{b}}$ diferente de GCRO $(\mathrm{P} \leq 0,05)$

Os estudos já realizados com roedores demonstram que o peptídeo presente no veneno crotálico ou a crotalfina apresenta importante efeito antinociceptivo, já comprovado em estudos que empregaram modelo de dor neuropática induzida pela constrição crônica do nervo isquiático (GUTIERREZ, 
2005) e em modelo de dor oncológica induzida experimentalmente (BRIGATTE et al., 2004), ambos com roedores. Nos estudos com dor inflamatória, o efeito antinociceptivo do veneno crotálico ou da crotalfina foi confirmado no modelo das contorções abdominais induzidas pelo ácido acético (GIORGI et al., 1993) e no modelo de hiperalgesia induzida pela carragenina ou prostaglandina (PICOLO et al., 2000; PICOLO \& CURY, 2004). No modelo da placa quente, o veneno crotálico produziu efeito antinociceptivo por até cinco dias (BRIGATTE et al., 2001).

Nos estudos supracitados que avaliaram o efeito analgésico da crotalfina, já havia alguma injúria tecidual prévia ou, então, inflamação induzida. Isso justifica a efetividade da crotalfina, enquanto no presente estudo não se observou efeito significativo. Por ser um opioide, a crotalfina em tecido íntegro torna-se pouco efetiva, pois sua ação analgésica baseia-se na liberação de IL-10, um mediador que possui ação antiinflamatória (FIORENTINO et al., 1991; WALL MALEFYT et al., 1991), e na elevação do nível plasmático de glicocorticóides por meio da estimulação do eixo hipotálamo-hipófise-adrenal (CHISARI et al., 1998).

\section{CONCLUSÃO}

A crotalfina, a morfina, o U50-488H e a fenilbutazona não produzem efeito antinociceptivo relevante em equinos submetidos à estimulação térmica em pele íntegra.

\section{AGRADECIMENTOS}

Ao Sr. Wiliiam Marandola, representante do COINFAR, pela doação de crotalfina. À FAPESP (processo n.05/04480-0) e ao CNPq (processo n.140038/2006-0) pelo suporte financeiro.

\section{FONTE DE AQUISIÇÃO}

a - Dolorimeter model 331 - IITC Inc. Life Science Instruments, USA

\section{COMITÊ DE ÉTICA E BIOSSEGURANÇA}

Este estudo foi aprovado pelo Comitê de Ética e Bem-estar Animal da FCAV/UNESP - Jaboticabal (processo n.009974-05).

\section{REFERÊNCIAS}

ANTONIJEVIC, I. et al. Perineural defect and peripheral opioid analgesia in inflammation. Journal of Neuroscience, v.15, p.165-172, 1995. Disponível em: <http://www.jneurosci.org/ content/15/1/165.full.pdf+html>. Acesso em: 01 maio, 2012. doi: 10.1136/adc.2003.032003.
BOATWRIGHT, S.E. et al. A comparison of N-butylscopolammonium bromide and butorphanol for analgesia using a balloon model of abdominal pain in ponies. Canadian Journal of Veterinary Research, v.60, p.65-68, 1996.

BRIGATE, P. et al. Whole Crotalus durissus terrificus snake venom induces antinociception in a rat model of cancer pain and crotoxin inhibits tumor growth. In: INTERNATIONAL SYMPOSIUM OF NITRIC OXIDE, CYTOKINES AND INFLAMMATION, 2004, Rio de Janeiro, RJ. Proceedings... Rio de Janeiro: International Symposium of Nitric Oxide, Cytokines and Inflammation, 2004. p.127.

BRIGATTE, P. et al. Tolerance to the antinociceptive effect of Crotalus durissus terrificus snake venom in mice is mediate by pharmacodynamic mechanisms. Toxicon, v.39, p.1399-1410, 2001. Disponível em: <http://www.sciencedirect.com/science/ article/pii/ S004101010100099X>. Acesso em: 01 maio, 2012.

CARREGARO, A.B. et al. Effects of buprenorphine on nociception and spontaneous locomotor activity in horses. American Journal of Veterinary Research, v.68, p.246-250, 2007. Disponível em: http://avmajournals.avma.org/doi/pdf/10.2460/ajvr.68.3.246. Acesso em: 01 ago, 2012. doi: 10.2460/ajvr.68.3.246.

CHISARI, A. et al. A phospholipase $\mathrm{A}_{2}$-related snake venom (from Crotalus durissus terrificus) stimulates neuroendocrine and immune functions: determination of different sites of action. Endocrinology, v.139, p.617-625, 1998. Disponível em: <http:// endo.endojournals.org/content/139/2/617.full.pdf $+\mathrm{html}>$. Acesso em: 01 maio, 2012. doi: 10.1210/en.139.2.617.

DHANJAL J.K. et al. Intravenous tramadol: effects, nociceptive properties, and pharmacokinetics in horses. Anaesthesia and Analgesia, v.36, p.581-90, 2009. Veterinary

DHAWAN, B.N. et al. Classification of opioid receptors. Pharmacological Reviews, v.48, p.567-592, 1989.

DICKENSON, A.H.; SULLIVAN, A.F. Evidence for a role of the NMDA receptor in the frequency dependent potentiation of deep rat dorsal horn nociceptive neurons following $\mathrm{C}$ fibre stimulation. Neuropharmacology, v.26, p.1235-1238, 1987. Disponível em: <http://ac.els-cdn.com/0028390887902759/1s2.0-0028390887902759-main.pdf?_tid=1adc 19b457e03342e801 00c2db822103\&acdnat=1343151465_9a9739443f54d17e22838f4 f9229df1b>. Acesso em: 01 maio, 2012.

DUKE, M.A. et al. Paradoxical effects of kappa-opioid stimulation on the locomotor activity and Fos immunoreactivity of the prewealing rat: role of dopamine receptors. Behavioral Neuroscience, v.111, p.1114-1422, 1997. Disponível em: <http:// psycnet.apa.org/journals/bne/111/5/1114.pdf >. Acesso em: 19 mar. 2012.

FIORENTINO, D.F. et al. IL-10 inhibits cytokine production by activated macrophages. Journal of Immunology, v.147, p.3815-3822, 1991. Disponível em: <http://www.jimmunol.org/ content/147/11/3815.full.pdf+html>. Acesso em: 01 maio, 2012.

GAVERIAUX-RUFF, C.; KIEFFER, B. Opioid receptors: gene structure and function. In: STEIN, C. Opioids in pain control - basic and clinical aspects. Cambridge: Cambridge University, 1999. p.1-21.

GIORGI, R. et al. Analgesic effect evoked by low molecular weight substances extracted from Crotalus durissus terrificus 
venom. Toxicon, v.31, p.1257-1265, 1993. Disponível em: <http:// www.sciencedirect.com/science/article/pii/0041010193903994>. Acesso em: 01 maio, 2012.

GUIRRO, E.C.B.P. Efeitos comportamental, clínico e antinociceptivo da crotalfina em equinos: comparação com morfina, U50-488H e fenilbutazona. 2008. 109f. Tese (Doutorado em Cirurgia Veterinária - Anestesiologia Veterinária) - Faculdade de Ciência Agrárias e Veterinárias, Universidade Estadual Paulista, Jaboticabal, SP.

GUIRRO, E.C.B.P. et al. Efeitos clínicos e comportamentais promovidos pela injeção intravenosa de morfina em equinos Puro Sangue Árabe. Ciência Animal Brasileira, v.11, n.4, p.853-859, 2010. Disponível em: <http://www.revistas.ufg.br/index.php/ vet/article/view/3116/8359>. Acesso em: 05 maio, 2012. doi: 10.5216/cab.v11i4.3116.

GUTIERREZ, V.P. Efeito do veneno de Crotalus durissus terrificus sobre a dor neuropática e a resposta imune. 2005 . 146f. Dissertação (Mestrado em Ciências - Farmacologia) Instituto de Ciências Biomédicas, Universidade de São Paulo, SP.

KALPRAVIDH, M. et al. Effects of butorphanol, flunixin, levorphanol, morphine, and xylazine in ponies. American Journal of Veterinary Research, v.45, n.2, p.217-223, 1984.

KAMERLING, S. et al. Dose related effects of the kappa agonist $\mathrm{U}-50,488 \mathrm{H}$ on behavior, nociception and autonomic response in the horse. Equine Veterinary Journal, v.20, n.2, p.114118, 1988. Disponível em: <http://onlinelibrary.wiley.com/ doi/10.1111/j.2042-3306.1988.tb01471.x/abstract>. Acesso em: 12 maio, 2012. doi: 10.1111/j.2042-3306.1988.tb01471.x.

KAMERLING, S.G. et al. A method for studying cutaneous pain perception and analgesia in horses. Journal of Pharmacological Methods, v.13, p.267-274, 1985. Disponível em: <http://www. sciencedirect.com/science/article/pii/0160540285900270>. Acesso em: 03 mar. 2012

LEMONICA, L.; PEREIRA, S. M. Dor: bases anátomo-fisiológicas e do tratamento. In: BRAZ, J. R. C.; CASTIGLIA, Y. M. M. Temas de Anestesiologia. Botucatu: Unesp, 1992. p.163-170.

LIKAR, R. et al. Efficacy of peripheral morphine analgesia in inflamed, non-inflamed and perineural tissue of dental surgery patients. Journal of Pain and Symptom Management, v.21, p.330-337, 2001. Disponível em: <http://www.sciencedirect.com/ science/article/pii/S0885392401002512〉. Acesso em: 23 jul. 2012.

LOVE. E.J. et al. Thermal and mechanical nociceptive threshold testing in horses: a review. Veterinary Anaesthesia and Analgesia, v.38, n.1, p.3-14, 2011. Disponível em: <http://onlinelibrary.wiley. com/doi/10.1111/j.1467-2995.2010.00580.x/pdf >. Acesso em: 01 ago, 2001. doi: 10.1111/j.1467-2995.2010.00580.x.

MAY, S.A. Anti-inflammatory agents. In: ROBINSON, N.E. Current therapy in equine medicine. 3.ed. Philadelphia: Saunders, 1992. p.14-18

MILlAN, M.J. Multiple opioid systems and pain. Pain, v.27, p.303-347, 1986. Disponível em: <http://www.sciencedirect. com/science?_ob=ArticleListURL\&_method=list\&_ ArticleListID $=1977798738 \&$ \&ort $=r \&$ st $=13 \&$ view $=c \&$ _ acct $=$ C000228598\&_version $=1 \&$ _urlVersion $=0$ \&_userid $=10 \&$ md5=1ebd080e368129b19eeada $8578 b 468$ fe \&searchtype=a $>$. Acesso em: 01 maio, 2012.

MITTEN, L.A.; HINCHCLIFF, K.W. Nonesteroidal antiinflammatory drugs. In: ROBINSON, N.E. Current therapy in equine medicine. 4.ed. Philadelphia: Saunders, 1997. p.724-727.

MUIR III, W.W. et al. Fármacos usados na medicação pré anestésica. In: ___ Manual de anestesia veterinária. 3.ed. Porto Alegre: Artmed, 2001. p.31-44.

NUNES, F.P.B. et al. Long-lasting anti-inflammatory properties of Crotalus durissus terrificus snake venom in mice. Toxicon, v.49, p.1090-1098, 2007. Disponível em: <http://www.sciencedirect. com/science?_ob=ArticleListURL\&_method=list\&_ ArticleListID $=1977800046 \&$ \&sort $=r \&$ st $=13 \&$ view $=c \&$ _ acct $=C 000228598$ \&_version $=1 \&$ _urlVersion $=0$ \&_userid $=10 \& \mathrm{md}$ 5=3d6da86e2d6a46887c08bf12df68e37e\&searchtype=a>. Acesso em: 01 maio, 2012.

PICOLO, G. et al. Activation of peripheral ATP-sensitive $\mathrm{K}^{+}$ channels mediates the antinociceptive effect of Crotalus durissus terrificus snake venom. European Journal of Pharmacology, v.469, p.57-64, 2003. Disponível em: <http://www.sciencedirect. com/science?_ob=ArticleListURL\&_method=list\&_ ArticleListID $=1977770867 \&$ _sort $=r \& \_s t=13 \&$ view $=c \&$ _ acct $=$ C000228598\&_version $=1 \& \_u r l$ Version $=0$ \&_userid $=10 \& \mathrm{md}$ $5=7 \mathrm{cb} 5 \mathrm{bf} 168219$ ef 1 fb769121edaa2b277\&searchtype $=\mathrm{a}>$. Acesso em: 01 maio, 2012.

PICOLO, G. et al. delta-Opioid receptors and nitric oxide mediate the analgesic effect of Crotalus durissus terrificus snake venom. European Journal of Pharmacology, v.391, p.55-62, 2000. Disponível em: <http://www.sciencedirect. com/science? ob=ArticleListURL\&_method=list\& ArticleListID $=1977774193 \&$ _sort $=r \&$ rst $=13 \&$ view $=c \&$ _ acct $=$ C000228598\&_version $=1 \&$ \&urlVersion $=0 \& \_u s e r i d=10 \& m$ $\mathrm{d} 5=22841 \mathrm{ccfd} 6 \mathrm{faa} 2 \mathrm{fc} 2 \mathrm{daa} 107 \mathrm{f} 3 \mathrm{f} 0 \mathrm{~b} 91 \mathrm{fb} \&$ searchtype $=\mathrm{a}>$. Acesso em: 01 maio, 2012.

PICOLO, G. et al. The antinociceptive effect of Crotalus durissus terrificus snake venom is mainly due to supraspinally integrated response. Toxicon, v.36, p.223-227, 1998. Disponível em: <http://www.sciencedirect.com/science?_ob=ArticleListURL\&_ method=list\&_ArticleListID $=1977773329 \&$ \&t $=13 \&$ view $=c \&$ _ acct $=$ C000228598\&_version $=1$ \&_urlVersion $=0$ \&_userid $=76936$ c5459ef2775010be4bc938fc3d5\&searchtype $=a>$. Acesso em: 01 maio, 2012.

PICOLO, G.; CURY, Y. Peripheral neuronal nitric oxide synthase activity mediates the antinociceptive effect of Crotalus durissus terrificus snake venom, a d- and $\kappa$-opioid receptor agonist. Life Sciences, v.75, p.559-573, 2004. Disponível em: <http://www. sciencedirect.com/science/article/pii/S0024320504002929>. Acesso em: 01 maio, 2012.

PIPPI, N.L. et al. A model for evaluating pain in ponies. Journal of Equine Medicine Surgery, v.3, p.430-435, 1979.

SCHULTZ, J.E.J.; GROSS, G.J. Opioids and cardioprotection. Pharmacology \& Therapeutics, v.89, p.123-137, 2001. Disponível em: <http://www.sciencedirect.com/science/article/pii/ S0163725800001066>. Acesso em: 01 maio, 2012.

SINATRA, R.S. Spinal and epidural opioids. In: ROGERS, M.C. et al. Principles and practice of anesthesiology. St. Louis: Mosby-Year Book, 1993. p.1425-1443. 
SKARDA,R.T.;MUIRIII,W.W. Comparisionofelectroacupuncture and butrophanol on respiratory and cardiovascular effects and rectal pain after controlled rectal distention in mares. American Journal of Veterinary Research, v.64, p.137-144, 2003.

SOUSA E SILVA, M.C.C. et al. The venom of South American ratllesnake inhibits macrophage functions and is endowed with anti-inflammatory properties. Mediators of Inflammation, v.5, p.18-23, 1996. Disponível em: <http://www.ncbi.nlm.nih.gov/ pmc/articles/PMC2365764/pdf/MI-05-018.pdf〉. Acesso em: 01 maio, 2012. doi: 10.1155/S0962935196000038.

STEIN, C. Peripheral mechanisms of opioid analgesy. Anesthesia and Analgesia, v.76, p.182-191, 1993. Disponível em: <http:// www.anesthesia-analgesia.org/content/76/1/182.full.pdf+html >. Acesso em: 01 maio, 2012.
STEIN, C.; LANG, L.J. Peripheral mechanisms of opioid analgesia. Current Opinion of Pharmacology, v.9, p.3-8, 2009. Disponível em: <http://www.sciencedirect.com/science/article/pii/ S1471489208002075>. Acesso em: 01 maio, 2012.

THURMON, J.C. et al. Preanesthetics and anesthetic adjunts. In: ___ Lumb \& Jones' veterinary anesthesia. Maryland: Williams \& Wilkins, 1996. p.183-210

TRUONG, W. et al. Mu opioid receptors and analgesia at a site of a peripheral nerve injury. Annals of Neurology, v.53, p.366375, 2003. Disponível em: <http://onlinelibrary.wiley.com/ doi/10.1002/ana.10465/abstract;sessionid=FF1ED750D84E16 17F2A67E9AC820D3E9.d02t03>. Acesso em: 01 maio, 2012. doi: 10.1002/ana.10465. 